\title{
Mobile sensing in Aedes aegypti larva detection with biological feature extraction
}

\author{
Dia Bitari Mei Yuana ${ }^{1}$, Wahjoe Tjatur Sesulihatien ${ }^{2}$, Achmad Basuki $^{3}$, Tri Harsono ${ }^{4}$, \\ Akhmad Alimudin ${ }^{5}$ Etik Ainun Rohmah ${ }^{6}$ \\ 1,2,3,4,5 Department of Electrical and Computer Engineering, Politeknik Elektronika Negeri Surabaya, Indonesia \\ ${ }^{6}$ Institute of Tropical Disease, Entomology laboratory Kampus C Universitas Airlangga, Indonesia
}

\section{Article Info}

Article history:

Received Aug 24, 2019

Revised Oct 27, 2019

Accepted Dec 11, 2019

\section{Keywords:}

Aedes aegypti larva

Biological feature extraction

Detection

Mobile sensing

\begin{abstract}
According to WHO, Dengue fever is the most critical and most rapidly mosquito-borne disease in the world over 50 years. Currently, the presence and detection of Aedes aegypti larvae (dengue-mosquitoes vector's) are only quantified by human perception. In large-scale data, we need to automate the process of larvae detection and classification as much as possible. This paper introduces the new method to automate Aedes larvae. We use Culex larva for comparison. This method consists of data acquisition of recorded motion video, spatial movement patterns, and image statistical classification. The results show a significant difference between the biological movements of Aedes aegypti and Culex under the same environmental conditions. In 50 videos consisting of 25 Aedes larvae videos and 25 Culex larvae videos, the accuracy was $84 \%$.
\end{abstract}

This is an open access article under the CC BY-SA license.

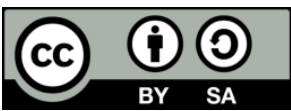

\section{Corresponding Author:}

Dia Bitari Mei Yuana,

Department of Electrical and Computer Engineering,

Politeknik Elektronika Negeri Surabaya,

Jl. Raya ITS, Sukolilo, Surabaya, 60111, Indonesia

Email: diabitari@pasca.student.pens.ac.id

\section{INTRODUCTION}

Aedes aegypti mosquito is a vector that transmits the Dengue virus from one person to another. The WHO says dengue is the most critical disease and most quickly transmitted through the Aedes aegypti mosquito. Due to the rapid spread of Dengue fever, the presence of mosquitoes plays an important role [1]. The difficulty in identifying Aedes vector ones is because their physical performance of all mosquitoes is similar [2]. Several studies on mosquito identification have been carried out, such as by molecular identification through DNA, morphological identification by sampling breeding sites [3], physical identification by image processing [4, 5], or by spatial similarity in several regions [6, 7]. However, it is difficult to implement their methods because they are expensive and require special expertise. Meanwhile, to combat Dengue fever, we need to empower ordinary people who don't have much money and are not very skilled in technology [8-10]. Therefore, we need to find a method that is simple in implementation, relatively inexpensive but accurate enough to classify larval [11, 12].

This paper discusses the method for classifying Aedes aegypti larvae. As a comparison, we utilize Culex, the common mosquito that lives in Indonesia [13]. Both have the same performance but different impacts: Aedes aegypti is very dangerous while Culex is not [14]. This method utilizes video captured by a standard camera. It is hoped to be useful to increase awareness of vector-borne diseases. We use the optical flow method to detect larval motion. Aedes larvae movements can be recognized by the optical flow method. A consistent pattern was analyzed using the optical flow method to detect Aedes larvae. Then, we use the Euclidean distance calculation to compare motion of the Aedes larvae and Culex larvae. 


\section{RESEARCH METHOD}

Larvae are one stage of the mosquito life cycle where mosquito eggs emerge in the water. In this stage, larvae spend most of their time feeding and growing [1]. Larvae can detect quick changes in light and when a shadow is cast on the water, they will defensively dive to the bottom of the water. It can also detect sudden vibrations in the water and will likewise dive down $[15,16]$.

To get food and grow, the biological behavior of mosquitoes moves from one point to another with a certain pattern. Each species in the mosquito family has a different pattern [17]. Therefore, an important feature for classifying larvae is the pattern of larval movement. In this paper, we focus on analyzing the movement of single larvae. We use video processing to recognize patterns of differences between Aedes and Culex. Figure 1 shows the diagram block of this method. Using cellular devices mounted on a tripod, the researchers recorded the movement of Aedes larvae and Culex larvae and then selected 15 frames to process.

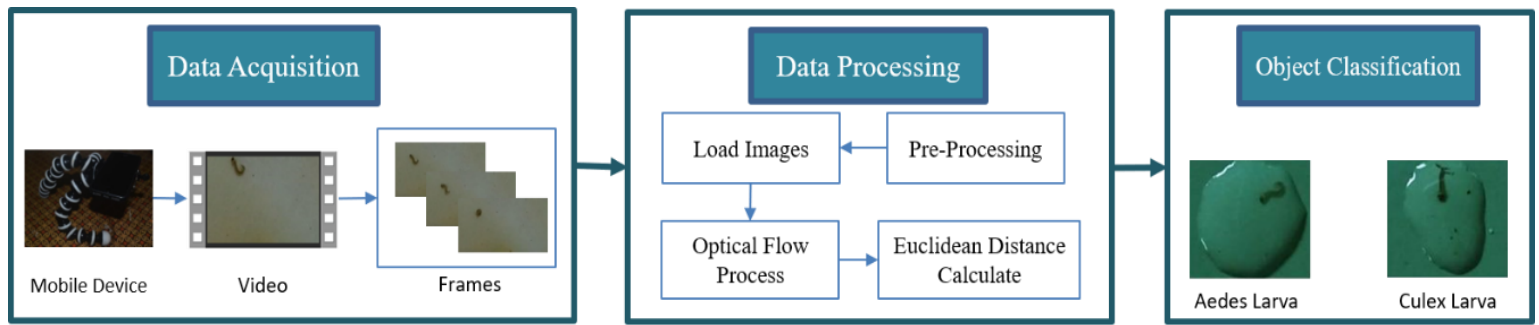

Figure 1. Diagram block of the method

Optical flow method can describe the meaning of motion [18]. To capture the movement, we should process video data. Movement patterns can be captured from moving objects in the video. This pattern is utilized to describe the type of object is moving. Figure 1 shows diagram block of the method to process dataset of Aedes aegypti and Culex. The dataset is taken using data collection techniques as data acquisition. Furthermore, the data is processed for further processing using the optical flow method. In optical flow, the differences movement patterns of Aedes and Culex larvae are analyzed. Aedes larvae have more consistent pattern compared to Culex larvae which unpredictable movement patterns. So, to recognize the movement patterns of Aedes larvae or Culex larvae, the researcher use the Euclidean distance calculation in the optical flow method.

\subsection{Data preparation}

To analyze the pattern of larvae motion, we set up the video recording system to collect the data in Entomology Laboratory, Airlangga University Surabaya, Indonesia. The system consists of a smartphone with a flexible tripod stand and larvae container that contains water. Figure 2(a) shows the system for image acquisition. Figure 2(b) shows the larvae container. It is a specific apparatus to sterilize the environment so that the water suitable for larvae during the collecting data process. Two types of mosquito's egg were incubated in two different containers.

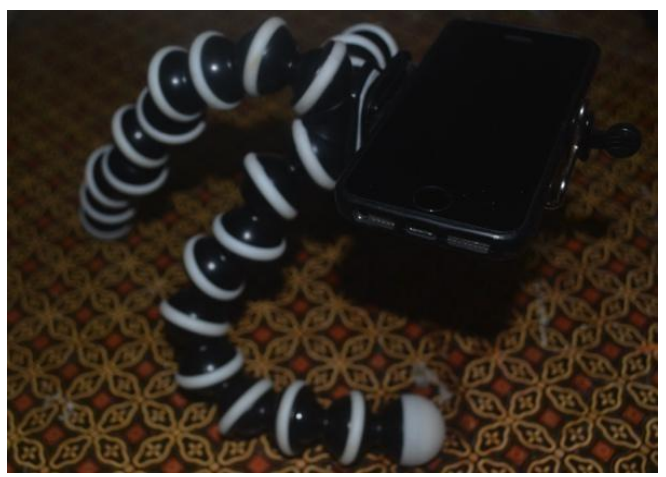

(a)

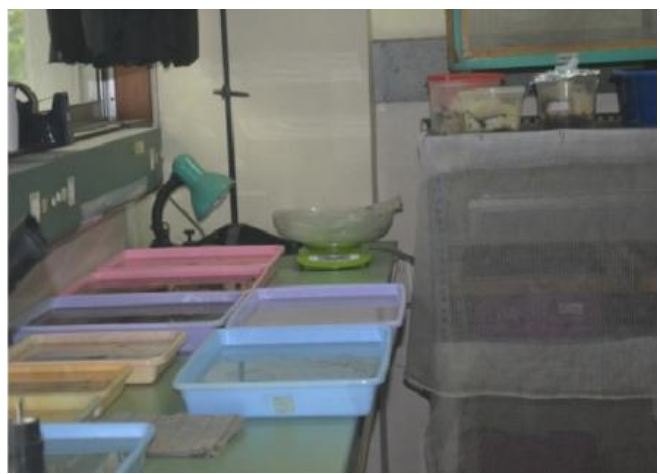

(b)

Figure 2. Materials and tools, (a) Image acquisition using mobile device, (b) Experimental setup 
Soon after the egg metamorphoses to be larvae, every larva is laid on the tray that contains water. Then we took a video of them during the larvae stage (7 days). Since our purpose is developing this software for people, and many of them are low-income, we use Ovo hand phone's camera: mobile device that low-income people in Indonesia usually have. We also set up the lighting as their home's lighting.

\subsection{Preliminary research}

Along with the literature study and gathered knowledge from the expert, we also conducted a preliminary study to convince our hypotheses about the biological characteristics of both mosquito types. We record 5 second's video data of Aedes aegypti and Culex, parsed into 5 frames and analyzed briefly. Figure 3 describes the pattern of motion serially of Aedes aegypti and Culex. In 5 second, there are differences in motion pattern between Aedes aegypti larvae and Culex larvae. Aedes aegypti larvae tends to move at Figure 3(a)-(e) while Culex tends to quiescent at Figure 3(f)-(j) frame 1 to frame 4 then moves in frame 5 . This preliminary study leads us to extract their features more

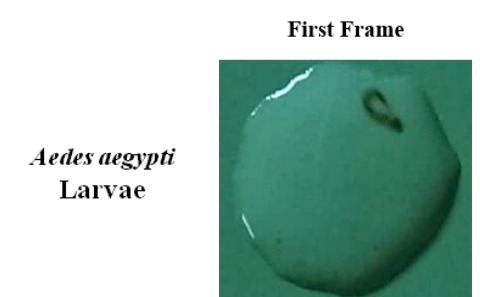

(a)

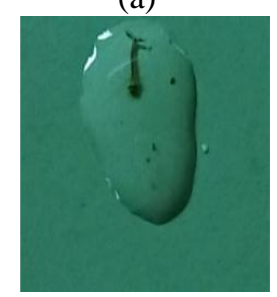

(f)

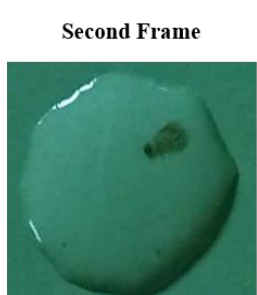

(b)

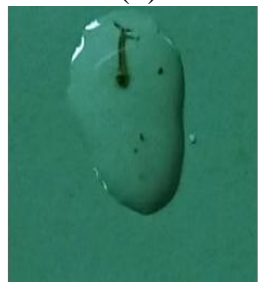

(g)

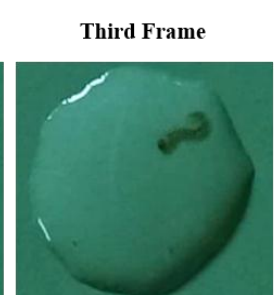

(c)

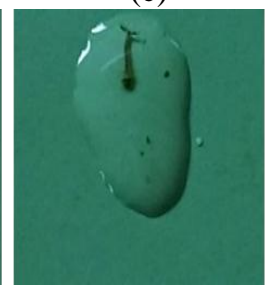

(h)

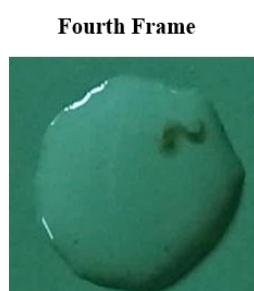

(d)

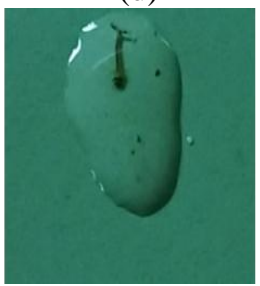

(i)

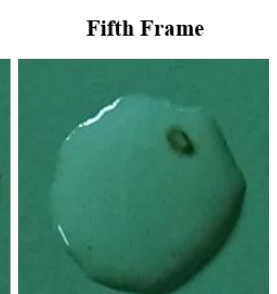

(e)

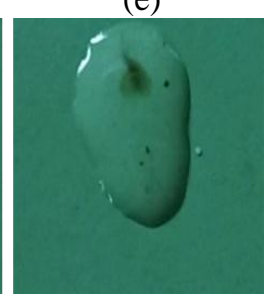

(j)

Figure 3. The frame of larva Aedes aegypti and Culex support, (a) Aedes aegypti larvae from first frame, (b) Aedes aegypti larvae from second frame, (c) Aedes aegypti larvae from third frame, (d) Aedes aegypti larvae from fourth frame, (e) Aedes aegypti larvae from fifth frame, (f) Culex larvae from first frame,

(g) Culex larvae from second frame, (h) Culex larvae from third frame, (i) Culex larvae from fourth frame,

(j) Culex larvae from fifth frame

\subsection{Preprocessing data}

Preprocessing data consist of parsing video data. In this research, 28 videos of Aedes aegypti's motion and 22 videos of Culex motion are captured. They are parsed into 15 frames and analyzed. The raw video data has a big resolution. It is not efficient for training. Therefore, we conducted pre-processing to avoid irrelevant images such as the background. Preprocessing data consist of:

- Converting video data to 15 image frames

- Converting the true color image rgb to the greyscale intensity image or frame

- Converting the image to integer

\subsection{Spatial movement pattern}

In disease spreading, contracting area and pattern of disease vector are important [18, 19]. To analyze spatial movement patterns, we employed an optical flow method. Optical flow is technique to detect, predict and interpret and compensate the movement of an object [15-20]. In this paper, we explore Lucas Canade algorithm to recognize the pattern of larvae [21, 22]. Larva's video is processed using optical flow. Each video is taken 15 frames for motion detection. The first process is finding the corners of each frame, and then using the Lucas Kanade concept by tracing the position of certain nodes in one frame to the next frame continuously with the image flow (velocity) vector (Vx, Vy) should fulfill (1) [23].

$$
\mathrm{I}_{\mathrm{x}}\left(q_{i}\right) V_{x}+\mathrm{I}_{\mathrm{y}}\left(q_{i}\right) V_{y}=\mathrm{I}_{\mathrm{t}}\left(q_{i}\right)
$$


Where Ix(qi), Iy(qi), It(qi) is partial derivatives to image I respect to the position of $x, y$ to time $t$ based on qi. In that sense, (1) is written in term matrix $A v=b$ as follows.

$$
A=\left[\begin{array}{c}
I_{x}\left(q_{1}\right) I_{y}\left(q_{1}\right) \\
I_{x}\left(q_{2}\right) I_{y}\left(q_{1}\right)
\end{array}\right] v=\left[\begin{array}{c}
V_{x} \\
\vdots \\
V_{y}
\end{array}\right] b=\begin{gathered}
-I_{t\left(q_{1}\right)} \\
I_{x}\left(q_{n}\right) I_{y}\left(q_{n}\right)
\end{gathered}
$$

As a result, the vector flow is a transpose matrix as shown in (3).

$$
A^{\mathrm{T}} \mathrm{Av}=\mathrm{A}^{\mathrm{T}} \mathrm{Ab}
$$

To get the spatial movement pattern, we perform several steps:

- Load image of 15 frames,

- Find the corners: starting from q+2 where $\mathrm{q}$ is $\mathrm{q}$ is called as an early object definition,

- Apply the Lucas Kanade method, and draw the optical flow vectors.

\subsection{Image statistical classification}

We employ Euclidean distance to recognize movement patterns of larvae. Value of Eucledian distance will high when the larvae move actively, vice versa. The total value of Euclidean distance was applied to detect the differences Aedes aegypti larvae and Culex larvae. In this case, we calculated Eucledian distance of every frame has a different matrix, total value of the matrix.

The Optical Flow method produces values of the movement in every frame [24]. Velocity of movement represents differences of motion pattern between Aedes larvae and Culex larvae. Theoretically, Aedes larvae move consistently while Culex larvae move inconsistently. Therefore, we can be conclude that the total velocity of Aedes larvae is higher than Culex larvae in early experimental (15 frame). The next step is calculated Euclidean distance from every frame with (4):

$$
\sqrt{\left(x_{n-} x_{n-1}\right)^{2}+\left(y_{n-} y_{n-1}\right)^{2}}
$$

where $x_{n}$ and $y_{n}$ are total matrix $x$ and $y$ at $\mathrm{t}=\mathrm{n}$, and $x_{n-1}$ dan $x_{n-1}$ are total matrix $x$ and $y$ at $\mathrm{t}=\mathrm{n}-1$.

\section{RESULTS AND ANALYSIS}

In this research, we captured 28 videos of Aedes aegypti and 14 videos of Culex in Laboratorium Entomology, Institute of Tropical Desease Airlangga University. Beftore analyzed the pattern, all video data were preprocessed as mention in section 2.3. The result is shown in Figure 4. Figure 4 shows a video sample used as test data. In one video that lasts 11 seconds produces 325 frames. However, researchers used 15 frames to be processed using the optical flow method to detect moving objects. After preprocessing, we loaded the image. This process includes removing irrelevant images such as small plankton or water contaminator. Then, find the corner. Every frame has a different matrix depend on the next object. The next step was calculated with Lucas Kanade method by (2)-(3).

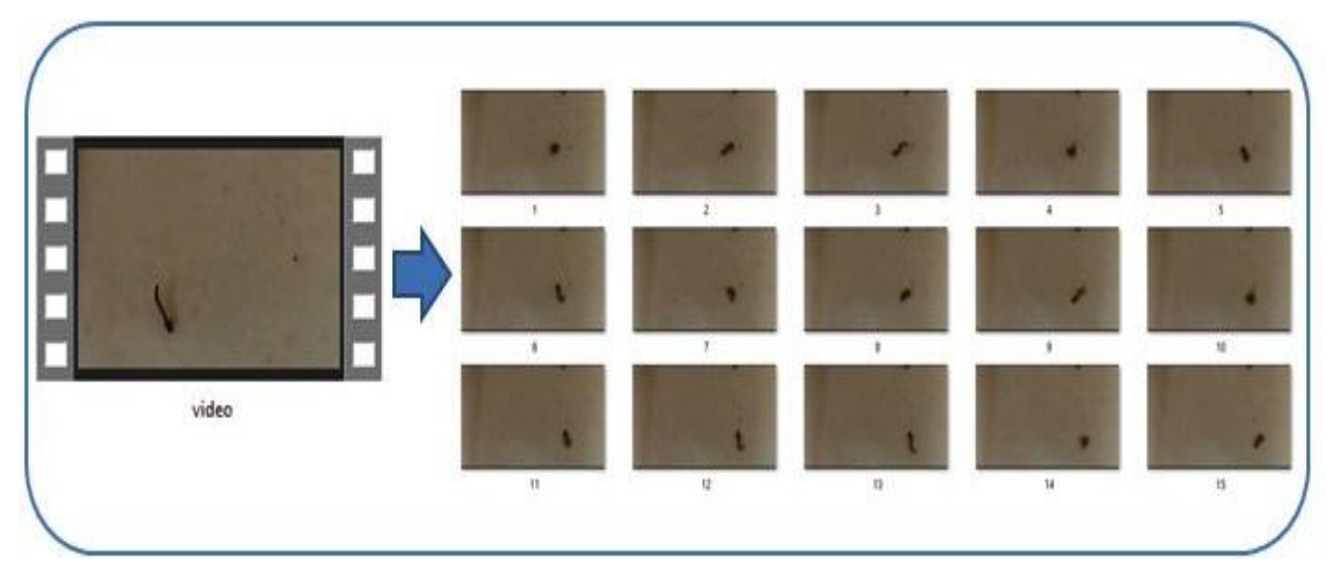

Figure 4. Result of preprocessing 
Based on matrix from 14 frames in find corner, process, discard corners was handled near the margins of the matrix frame. Thus, the moving object was reflected in the next frame by the optical flow vector calculated in Lucas Canade method. The result of the Lucas Kanade method is saved as a one-dimensional matrix. Every matrix may different as the number of the matrix in find corner also different. We found the differences vector optical flow that reflecting spatial objects movement from frame 1 to frame 14 of every video. To classify the larvae, Eucludean distance is applied. The distance is calculated from the next frame to the previous frame until 15 frames. From 15 frames, we got 13 values of Eucledian distance. Experiment, the results of different Euclidean distance is calculated.

Figure 5 shows 50 video experiments consisting of 25 Aedes Larvae videos and 25 Culex larvae videos. The blue color indicates the Euclidean Distance value of the Aedes larvae and the red color indicates the Euclidean distance value of the Culex larvae. The yellow line is the threshold of Aedes larvae and Culex larvae based on the average value of Euclidean distance. For Aedes larvae, the threshold is not more than one while Culex larvae is more than 1. The average Eucledean Distance of both Aedes and Culex was calculated. The result is shown in Table 1.

The Euclidean distance of Aedes larvae is 1.181372 while in Culex larvae is 0.060723 . It shows that the movement of Aedes larvae is relatively more consistent than Culex larvae. Details of the values of Euclidean distance of Culex larvae and Aedes larvae in Figure 5 are discussed in Table 2. The video numbers represent each Aedes larvae and Culex larvae that produce Euclidean distance values. Table 2 shows there are 25 videos with a maximum of 25 Aedes larvae videos and 25 Culex larvae videos so that the total video trials are 50 videos.

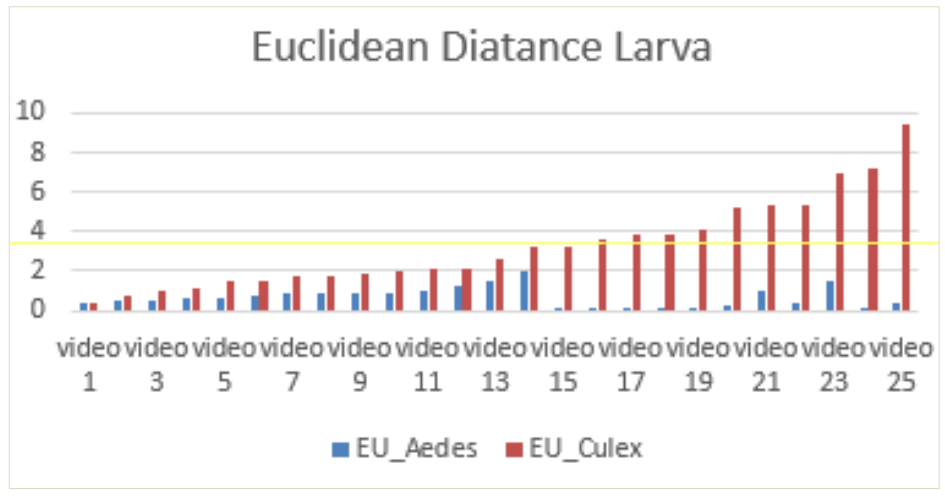

Figure 5. Euclidean distance larvae
Tabel 1. Euclidean distance Aedes

\begin{tabular}{lll}
\multicolumn{3}{c}{ and Culex frame by frame } \\
\hline Frame & Aedes & Culex \\
\hline $2-3$ & 2,70 & 0,08 \\
$2-3$ & 1,99 & 0,13 \\
$4-5$ & 0,98 & 0,03 \\
$5-6$ & 0,74 & 0,02 \\
$6-7$ & 0,68 & 0,06 \\
$7-8$ & 0,51 & 0,03 \\
$8-9$ & 0,71 & 0,09 \\
$9-10$ & 0,94 & 0,01 \\
$10-11$ & 0,92 & 0,02 \\
$11-12$ & 1,14 & 0,01 \\
$12-13$ & 1,46 & 0,09 \\
$13-14$ & 1,41 & 0,10 \\
$14-15$ & 1,10 & 0,06 \\
Average & 1,181372 & 0,060723 \\
\hline
\end{tabular}

Tabel 2. Euclidean distance of the larvae's Aedes

\begin{tabular}{lllll}
\hline Larva & EU_Aedes & Result & EU_Culex & Result \\
\hline video 1 & 0,389693 & TRUE & 0,3326 & FALSE \\
video 2 & 0,445714 & TRUE & 0,70682 & FALSE \\
video 3 & 0,49031 & TRUE & 0,95355 & FALSE \\
video 4 & 0,667548 & TRUE & 1,07183 & TRUE \\
video 5 & 0,676606 & TRUE & 1,47463 & TRUE \\
video 6 & 0,771291 & TRUE & 1,50798 & TRUE \\
video 7 & 0,827584 & TRUE & 1,78752 & TRUE \\
video 8 & 0,850508 & TRUE & 1,78752 & TRUE \\
video 9 & 0,884203 & TRUE & 1,86375 & TRUE \\
video 10 & 0,927365 & TRUE & 2,0422 & TRUE \\
video 11 & 0,992542 & TRUE & 2,07134 & TRUE \\
video 12 & 1,19805 & FALSE & 2,14131 & TRUE \\
video 13 & 1,495861 & FALSE & 2,55891 & TRUE \\
video 14 & 1,963727 & FALSE & 3,19357 & TRUE \\
video 15 & 0,058198 & TRUE & 3,22102 & TRUE \\
video 16 & 0,088696 & TRUE & 3,56586 & TRUE \\
video 17 & 0,126362 & TRUE & 3,83153 & TRUE \\
video 18 & 0,150237 & TRUE & 3,87637 & TRUE \\
video 19 & 0,165304 & TRUE & 4,14823 & TRUE \\
video 20 & 0,299907 & TRUE & 5,22371 & TRUE \\
video 21 & 1,05906 & FALSE & 5,32439 & TRUE \\
video 22 & 0,342779 & TRUE & 5,32846 & TRUE \\
video 23 & 1,544764 & FALSE & 7,01613 & TRUE \\
video 24 & 0,078225 & TRUE & 7,16964 & TRUE \\
video 25 & 0,338474 & TRUE & 9,46401 & TRUE \\
\hline
\end{tabular}


Based on the test results of 50 videos consisting of 25 videos of Aedes larvae and 25 videos of Culex larvae, there were five "FALSE" in e Aedes larvae and three in Culex larvae, as shown in Table 2. So, the accuracy of the testing system was $84 \%$. A comparison between both Euclidean distance of the two mosquitoes is shown in Figure 11. Regarding the influence of water quality in mosquito movements, in future research, we will emphasize the application of sensing fusion as applied to coral disease [25].

\section{CONCLUSION}

The biological feature extraction by using mobile sensing is conducted. The result shows that larvae detection using vision-based perception is powerful to replace chemical and morphological features. The accuracy of the system is $84 \%$. Also, it is easy to handle and user-friendly. However, there several challenges before mobile sensing is launched, such as the influence of water quality and movement patterns of crowd larvae. All should be handling by vision-based perception.

\section{ACKNOWLEDGEMENTS}

The authors would like to thank Ministry of Education Indonesia for supporting this research through University Flagship Research (PTUPT) funding.

\section{REFERENCES}

[1] World Health Organization, "Vector-borne disease," who.int, 2017, [Online], Available at: http://www.who.int/newsroom/fact-sheets/detail/vector-borne-diseases. [Accesed May. 08, 2019].

[2] A. Bar and J. Andrew, "Morphology and morphometry of Aedes aegypti larvae," Annual Research \& Review in Biology, vol. 3, no. 1, pp. 1-21, 2013.

[3] J. Batovska, M. Blacket, K. Brown, and S. E. Lynch, "Molecular identification of mosquitoes (Diptera: Culicidae) in Southeastern Australia,"Ecology and Evolution,vol. 6, no. 9, pp. 3001-3011, May 2016.

[4] A. M. Ahmed, "Mosquito autogeny in Aedes caspius (Diptera: Culicidae): Alterations of larval nourishments reservation upon bacterial infection," Insect Science, vol. 10, no. 4, pp. 472-484, 2013.

[5] S. McFeeters, "Using the normalized difference water index (NDWI) within a geographic information system to detect swimming pools for mosquito abatement: A practical approach," Remote Sensing, vol. 5, no. 7 pp. 3544-3561, 2013.

[6] A. Bruhn, J. Weickert, and C. Schnörr, "Lucas/Kanade meets Horn/Schunck: Combining local and global optic flow methods," International Journal of Computer Vision, vol. 61, no. 3, pp. 211-231, 2005.

[7] P. P. Samuel and B. K. Tyagi, "Diagnostic methods for detection \& isolation of dengue viruses from vector mosquitoes," Indian Journal of Medical Research, vol. 123, no. 5, pp. 615-628, 2016.

[8] T. Brox, A. Bruhn, N. Papenberg, and J. Weickert, "High accuracy optical flow estimation based on a theory for warping," European conference on Copturer Vision, pp 25-36, 2004.

[9] W. T. Sesulihatien, Y. Kiyoki, S. Sasaki, A. Safie, S. Yotopranoto, V. Sornlertlamvanich, and P. Chawakitchareon, "Building the vector-control collaborative strategy in dengue fever: Case Surabaya, Kuala Lumpur, Bangkok," Information Modelling and Knowledge Bases XXVIII, vol. 292, pp. 94-105, 2017.

[10] W. T. Sesulihatien and Y. Kiyoki, "A dengue location-contraction risk calculation method for analyzing diseasespread," Information Modelling and Knowledge Bases XXVI, vol. 272, pp. 396-407, 2017.

[11] N. Mayer, E. Ilg, P. Hausser, P. Fischer, D. Cremers, A. Dosovitskiy, and T. Brox, "A large dataset to train convolutional networks for disparity, optical flow, and scene flow estimation," 2016 IEEE Conference on Computer Vision and Pattern Recognition (CVPR), pp. 4040-4048, 2016.

[12] O. Mirat, J. R. Sternberg, K. E. Severi, and C. Wyart, "ZebraZoom: An automated program for high-throughput behavioral analysis and categorization," Frontiers in Neural Circuits, vol. 7, no. art. 107, pp. 1-12, 2013.

[13] W. T. Sesulihatien, S. Rungsupa, A. Hansuebai, P. Chawkitchareon, S. Sasaki, and Y. Kiyoki, "Event-Based feature extraction for analyzing the first damage of coral under temperature and light intensity stressor," 2016 International Conference on Knowledge Creation and Intelligent Computing (KCIC), pp. 113-119, 2016.

[14] S. Ningsih and A. Ribal, "An optimal integrated vector control for prevention the transmission of dengue," Journal of Physics: Conference Series, vol. 1245, no. 1, pp. 1-14, 2019.

[15] A. Ponlawat, J. G. Scott, and L. C. Harrington, "insecticide susceptibility of Aedes aegypti and Aedes albopictus across Thailand," Journal of Medical Entomology, vol. 42, no. 5, pp. 821-825, 2005.

[16] V. M. Margam, D. B. Gelman, and S. R. Palli, "Ecdysteroid titers and developmental expression of ecdysteroidregulated genes during metamorphosis of the yellow fever mosquito, Aedes aegypti (Diptera: Culicidae)," Journal of Insect Physiology, vol. 52, no. 6, pp. 558-568, 2016.

[17] J. S. Harding, C. Brown, F. Jones, and R. Taylor, "Distribution and habitats of mosquito larvae in the Kingdom of Tonga,” Australian Journal of Entomology, vol. 46, no. 4, pp. 332-338, 2007.

[18] J. Cho, Y. Jung, D. S. Kim, S. Lee, and Y. Jung, "Moving object detection based on optical flow estimation and a Gaussian mixture model for advanced driver assistance systems," Sensors, vol. 19, no. 4, pp. 1-14, 2019.

[19] R. Szeliski., "Computer vision: Algorithms and applications," Springer Science and Business Media, 2010. 
[20] J. J. Yu, A. W. Harley, and K. G. Derpanis, "Back to basics: Unsupervised learning of optical flow via brightness constancy and motion smoothness," European Conference on Computer Vision, pp. 3-10, 2016.

[21] C. Tomasi, "Detection and tracking of point features technical report CMU-CS-91-132," International Journal of Computer Vision, 1991.

[22] S. Aslani and H. Mahdavi-Nasab,"Optical flow-based moving object detection and tracking for traffic surveillance," Int. J. of Electrical, Comp., Energetic, Electronic and Comm. Eng., vol. 7, no. 9, pp. 1252-1256, 2013.

[23] R. Tosepu, D. S. Effendy, and H. Bahar, "Indonesian strategy in reducing Aedes aegypty diseases in ASEAN economic community era,” Int. J. Res. Med. Sc., vol. 3, no. 7, pp. 1578-1582, 2015.

[24] P. Khorrami, J. Wang, and T. Huang, "Multiple animal species detection using robust principal component analysis and large displacement optical flow," Proc. of the 21st Int. Conf. on Pattern Recognition (ICPR), pp. 11-15, 2012.

[25] A. Sanchez-Ortiz, A. Fierro-Radilla, A. Arista-Jalife, M. Cedillo-Hernandez, M. Nakano-Miyatake, D. RoblesCamarillo, and V. Cuatepotzo-Jiménez, "Mosquito larva classification method based on convolutional neural networks," Int. Conference on Electronics, Communications and Computers (CONIELECOMP), pp. 1-6, 2017.

\section{BIOGRAPHIES OF AUTHORS}

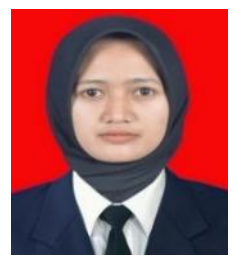

DiaBitari Mei Yuana received her first degree from Informatics Engineering and Computer Engineering Department, Politeknik Elektronika Negeri Surabaya (PENS), Indonesia 2017 and now she was a student of Applied Master Degree in Informatics Engineering and Computer Department. She acts as a part of Environmental Research Laboratory member since 2017. She was interested in Image Processing, Video Processing, Object Detection System, and Software development.

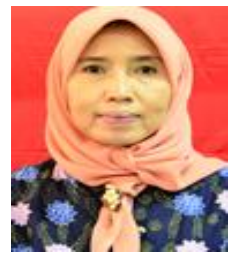

Ir. WahjoeTjaturSesulihatien, MT., Ph.D. is currently an teaches in Postgraduate and Electrical Engineering at PENS, Indonesia. She received first dgree from Institut Teknologi Sepuluh November in 1988 and her postgraduated program in 1999. Her PhD. Degree in Keio University, Japan in 2017. Her research interest is Environmental Modelling and Anaysis.

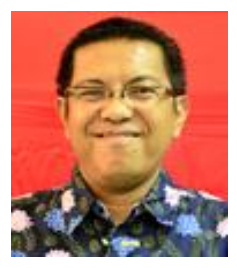

Drs. Achmad Basuki, M.Kom., Ph.D. is currently an teaches in Postgraduate of Computer and Informatics Technology at PENS, Indonesia. He received first degree of Mathematics at Institut Teknologi Sepuluh November in 1992 and master program of Informatics Engineering in 1999. His Ph.D. Degree in Saga University, Japan in 2011. His research interest is Computer Vision, Simulation and Modeling.

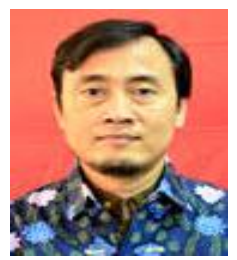

Tri Harsono, Ssi., M.Kom., Ph.D received his first degree from the Mathematics FMIPA and master degree from Informatic Engineering in Institut Teknologi Sepuluh Nopember (ITS). Then, he received Ph.D program from Computer Science, Saga University, Japan. The research interest in modelling and simulation. He is currently an teaches in Politeinik Elektronika Negeri Surabaya (PENS)

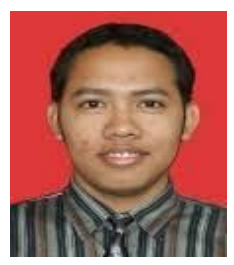

Akhmad Alimudin, S.ST., M.Kom is a lecturer in Politeknik Elektronika Negeri Surabaya (PENS). He heads the UPT Komputer at PENS.

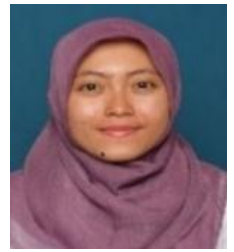

Etik Ainun Rohmah worked as a researcher in the Entolomology Laboratory of the Institute of Tropical Diseases (ITD) Surabaya, Indonesia in 2011 until now. One of the fields of study focus is a entomology, molecular biology, histology, and ecology. received her first degree from Dept. Biology, Faculty of Science and Technology, and magister of Tropical Medicine, Universitas Airlangga. 